\title{
Hemodynamic effects of vacuum-assisted closure therapy in cardiac surgery: Assessment using magnetic resonance imaging
}

\author{
Rainer Petzina, MD, ${ }^{a}$ Martin Ugander, MD, PhD, ${ }^{b}$ Lotta Gustafsson, MD, PhD, ${ }^{a}$ Henrik Engblom, MD, $\mathrm{PhD},{ }^{\mathrm{b}}$ \\ Johan Sjögren, $\mathrm{MD}, \mathrm{PhD}^{\mathrm{c}}$ Roland Hetzer, $\mathrm{MD}, \mathrm{PhD}{ }^{\mathrm{d}}$ Richard Ingemansson, $\mathrm{MD}, \mathrm{PhD},{ }^{\mathrm{c}}$ Håkan Arheden, $\mathrm{MD}, \mathrm{PhD},{ }^{\mathrm{b}}$ and \\ Malin Malmsjö, MD, PhD
}

From the Departments of Medicine, ${ }^{a}$ Clinical Physiology, ${ }^{\mathrm{b}}$ and Cardiothoracic Surgery, ${ }^{\mathrm{c}}$ Lund University Hospital, Lund, Sweden; German Heart Institute, ${ }^{\mathrm{d}}$ Berlin, Germany.

This study was supported by the Ander Otto Swärds Foundation/Ulrika Eklunds Foundation, Anna Lisa and Sven Eric Lundgrens foundation for medical research, Åke Wiberg Foundation, the M. Bergval Foundation, the Swedish Medical Association, the Royal Physiographic Society in Lund, the Swedish Medical Research Council, the Crafoord Foundation, the Swedish Heart-Lung Foundation, the Swedish Government Grant for Clinical Research, and the Swedish Hypertension Society.

Received for publication Sept 26, 2006; revisions received Jan 2, 2007; accepted for publication Jan 8, 2007.

Address for reprints: Malin Malmsjö, $\mathrm{MD}, \mathrm{PhD}$, Vascular Research, Lund University, BMC A13, SE-221 84 Lund, Sweden (E-mail: malin.malmsjo@med.lu.se).

J Thorac Cardiovasc Surg 2007;133:1154-62 $0022-5223 / \$ 32.00$

Copyright () 2007 by The American Association for Thoracic Surgery

doi:10.1016/j.jtcvs.2007.01.011
Objective: The hemodynamic effects of vacuum-assisted closure therapy in cardiac surgery are debated. The aim of the present study was to quantify cardiac output and left ventricular chamber volumes after vacuum-assisted closure using magnetic resonance imaging, which is known to be the most accurate method for quantifying these measures.

Methods: Six pigs had median sternotomy followed by vacuum-assisted closure treatment in the presence and absence of a paraffin gauze interface dressing. Cardiac output and stroke volume were examined using magnetic resonance imaging flow quantification (breath-hold and real-time). Chamber volumes were assessed using cine magnetic resonance imaging.

Results: Cardiac output and stroke volume decreased immediately after application of negative pressures of 75,125 , and $175 \mathrm{~mm} \mathrm{Hg}(13 \% \pm 1 \%$ decrease in cardiac output). Interposition of 4 layers of paraffin gauze dressing over the heart during vacuum-assisted closure therapy resulted in a smaller decrease in cardiac output $(8 \% \pm 1 \%)$.

Conclusions: Vacuum-assisted closure therapy results in an immediate decrease in cardiac output, although to a lesser extent than shown previously. Covering the heart with a wound interface dressing lessens the hemodynamic effects of vacuumassisted closure.

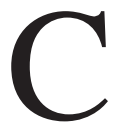
ardiac surgery is complicated by poststernotomy mediastinitis, a potentially life-threatening condition, ${ }^{1}$ in $1 \%$ to $5 \%$ of all procedures. ${ }^{2}$ The reported early mortality in poststernotomy mediastinitis after coronary bypass surgery grafting is between $8 \%$ and $25 \%,{ }^{3,4}$ Established treatment of poststernotomy mediastinitis includes surgical debridement, drainage, irrigation, and reconstruction using pectoral muscle flap or omentum transposition. In 1999, Obdeijn and colleagues ${ }^{5}$ described a new mode of treatment for poststernotomy mediastinitis using a vacuum-assisted closure (VAC) technique, which is based on the principle of applying subatmospheric pressure by controlled suction through a porous dressing. The technique, also known as topical negative pressure therapy, has resulted in excellent clinical outcome. ${ }^{6}$ Scientific evidence regarding the mechanisms by which VAC promotes wound healing has started to emerge, although knowledge of the effects on heart and lung function is still limited. The organs in the mediastinum are hemodynamically crucial, and both vulnerable bypass grafts and reduced cardiac function should be taken into consideration during VAC therapy of sternotomy wounds.

The hemodynamic effects of VAC therapy have become a matter of controversy. Conquest and colleagues ${ }^{7}$ used sonometry to show that subatmospheric pressure decreased the left ventricular volume and cardiac output in pigs by approximately $30 \%$. This could be prevented by rotating a rectus muscle flap over the mediastinal 


$$
\begin{aligned}
& \text { Abbreviations and Acronyms } \\
& \text { ECG }=\text { electrocardiogram } \\
& \mathrm{MRI}=\text { magnetic resonance imaging } \\
& \mathrm{SEM}=\text { standard error of the mean } \\
& \mathrm{TE}=\text { echo time } \\
& \mathrm{TR}=\text { repetition time } \\
& \mathrm{VAC}=\text { vacuum-assisted closure }
\end{aligned}
$$

wound. Conversely, in a study by Sjögren and associates ${ }^{8}$ using thermodilution, cardiac output was found not to be impaired by pressures between -50 and $-175 \mathrm{~mm} \mathrm{Hg}$. Instead, a slight increase in cardiac output was observed at $-75 \mathrm{~mm} \mathrm{Hg}$.

Magnetic resonance imaging (MRI) is known to be an accurate method for quantifying cardiac output and chamber volumes. ${ }^{9}$ The aim of the present study was to analyze the hemodynamic effects of VAC, including cardiac output and left ventricular volumes, using MRI. Furthermore, a common clinical practice is to place 4 layers of paraffin gauze dressing over the heart for protection. ${ }^{10}$ Therefore, we also aimed to examine the influence of an interface dressing on the effects of VAC on central hemodynamics.

\section{Materials and Methods}

Animals

A porcine sternotomy wound model was used. Six domestic landrace pigs with a mean body weight of $50 \mathrm{~kg}$ were put on a fast overnight with free access to water. The study was approved by the Ethics Committee for Animal Research, Lund University, Sweden. The investigation complied with the Guide for the Care and Use of Laboratory Animals as recommended by the U.S. National Institutes of Health. ${ }^{11}$ The anesthesia was induced and the surgical procedure was performed as previously described. ${ }^{2}$

\section{VAC Technique}

The surgical technique for VAC application has been described in detail previously. ${ }^{10}$ Four layers of paraffin gauze dressing were placed at the bottom of the wound to protect the right ventricle and lung tissue from the sternal edges. Sterile polyurethane foam dressing was placed between the sternal edges. A second layer of foam was placed subcutaneously and secured with a running suture to the surrounding skin. Drainage tubes were inserted into the foam. The wound was then sealed with a transparent adhesive drape. The drainage tubes were connected to a purpose-built vacuum pump and a canister for collection of effluents. The vacuum source (VAC pump unit, KCI, Austin, Tex) was set to deliver a continuous pressure of $-75,-125$, or $-175 \mathrm{~mm} \mathrm{Hg}$. For illustration, see Figure 1.

\section{Hemodynamic Assessment}

Cardiac output and left ventricular volumes were examined using MRI. Baseline measurements were recorded when the VAC source was turned off, and the effect of VAC was examined at 3 different negative pressures $(75,125$, and $175 \mathrm{~mm} \mathrm{Hg})$. After 1 series of

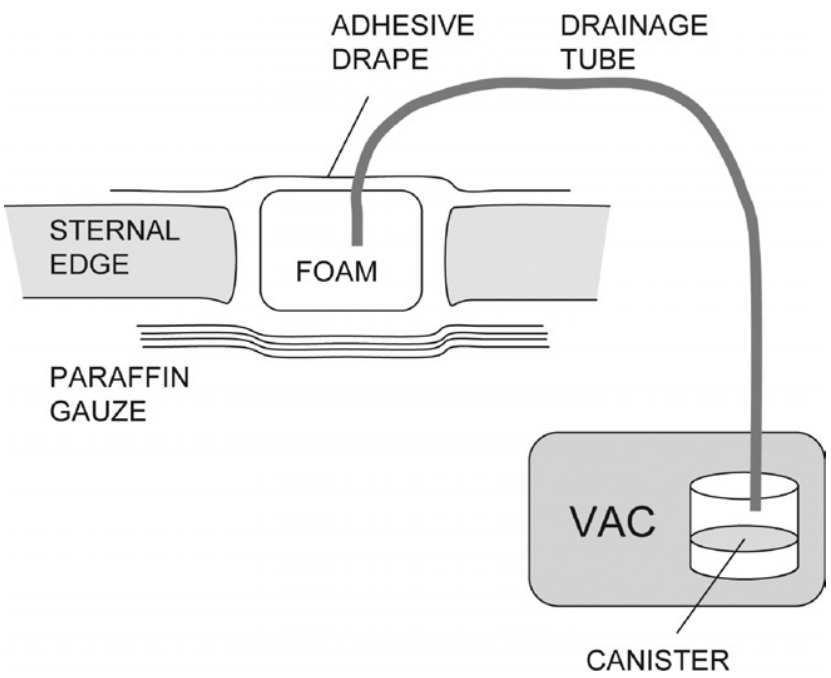

Figure 1. Cross section of the thoracic cavity with an open sternotomy. The heart is covered with 4 layers of paraffin gauze dressing. A polyurethane foam dressing is placed between the sternal edges, and noncollapsible drainage tubes are inserted into the foam. The open wound is sealed with transparent adhesive drape, and drainage tubes are connected to a purpose-built vacuum source.

cardiac output and left ventricular volume measurements, the wound interface dressing was removed and a new series of measurements were performed using identical settings.

\section{MRI Flow Quantification of Cardiac Output With an Established technique}

Cardiac output was assessed using MRI flow quantification. The established technique for MRI flow quantification involves data acquisition in the ascending aorta during typically 15 consecutive heartbeats during end-expiratory breath hold, yielding the instantaneous flow for 40 time points over 1 cardiac cycle. This technique has high spatial resolution and high temporal resolution within the cardiac cycle and reflects the average flow during 15 heartbeats.

Cardiac output experiments were performed with this technique in 6 pigs to measure cardiac output with maximal accuracy. Two measurements were performed immediately before the negative pressure was turned on ( 0 minutes) and then $0.5,1,1.5,2,2.5$, and 3 minutes after application of negative pressure. Measurements were also performed 1.5 and 2 minutes after the negative pressure had been switched off. Mean values were calculated from the 2 measurements before and the 2 measurements after negative pressure application and are referred to as "VAC off" or "baseline."

\section{MRI Flow Quantification of Cardiac Output in Real-time}

Cardiac output was also quantified continuously using a novel real-time MRI flow quantification technique. The real-time technique involves continuous quantification of the instantaneous flow in the ascending aorta with typically 20 measurements per cardiac 
cycle. Compared with the established technique, the real-time technique has a lower spatial resolution and a lower temporal resolution within the cardiac cycle. However, the real-time technique makes it possible to continuously assess cardiac output for each heartbeat over a given period of time.

Real-time flow quantification was performed in 2 pigs to illustrate the initial time course of events with maximal temporal resolution. Imaging was undertaken at the 3 different pressures $(-75,-125,-175 \mathrm{~mm} \mathrm{Hg})$ with and without the presence of wound interface dressing. The vacuum source was turned on 5 seconds after the start of real-time imaging and was continued for a total of 44 seconds. Images were assessed visually to determine when the lateral movement of the sternal edges by VAC was completed. This was presumed to reflect the time point when the negative pressure had reached a steady state.

\section{Imaging Techniques}

All MRI was performed using a 1.5-T scanner (Philips Intera, Best, The Netherlands). MRI flow quantification was undertaken either using the established technique (during breath-hold) or using realtime imaging during free breathing. Breath-hold acquisition was performed using a retrospectively electrocardiogram (ECG)-triggered turbo field echo phase-contrast velocity-encoded sequence. Typical imaging parameters for breath-hold acquisition were: acquired spatial resolution $=2.3 \times 2.3 \times 10 \mathrm{~mm}$, repetition time $(\mathrm{TR})=4.1 \mathrm{~ms}$, echo time $(\mathrm{TE})=2.4 \mathrm{~ms}$, velocity-encoding gradient $=200 \mathrm{~cm} / \mathrm{s}$, sensitivity encoding factor $($ SENSE $)=2$.

Flow quantification was undertaken using a real-time sequence during free breathing. Typical imaging parameters were: ECG triggering $=$ no, acquired spatial resolution $=3.4 \times 3.5 \times 12 \mathrm{~mm}$, $\mathrm{TR}=20.6 \mathrm{~ms}, \mathrm{TE}=6.8 \mathrm{~ms}$, velocity encoding gradient $=150$ $\mathrm{cm} / \mathrm{s}$, SENSE factor $=2.5$, echo planar imaging factor $=37$, duration of acquisition $=42 \mathrm{~s}$. Flow quantification was undertaken with automatic vessel segmentation and manual adjustments using software provided by the vendor (Viewforum, Philips, Best, The Netherlands). The cardiac cycle duration and heart rate for realtime imaging was determined as the difference in time between the image frame for onset of the systolic increase in aortic flow and the last time frame before onset of the next cardiac cycle. Cardiac output was calculated as the product of stroke volume and heart rate.

\section{MRI Quantification of Left Ventricular Volumes}

The left ventricular chamber volumes were quantified using a retrospectively ECG-triggered cine-balanced turbo field echo sequence. Typical imaging parameters were: acquired spatial resolution $=2.3 \times 2.9 \times 10 \mathrm{~mm}, \mathrm{TR}=2.9 \mathrm{~ms}, \mathrm{TE}=1.4 \mathrm{~ms}$, SENSE factor $=2$, heart phases $=30$. Measurements were performed using freely available software (Segment 1.350, http://segment. heiberg.se/) and previously described techniques. ${ }^{13}$ One baseline measurement was performed just before the negative pressure was turned on, and 1 measurement was performed 1.5 minutes after the negative pressure $(75,125$, or $175 \mathrm{~mm} \mathrm{Hg})$ had reached target level.

\section{Wound Negative Pressure Measurements}

The influence of paraffin gauze interface dressings on the delivery of topical negative pressure therapy to the wound was examined.
The vacuum source was set to successively deliver negative pressures from 50 to $200 \mathrm{~mm} \mathrm{Hg}$, at $25 \mathrm{~mm} \mathrm{Hg}$ increments. The tip of a saline solution-filled pressure catheter was placed in the space between the heart and the wound dressing. The pressure catheter was connected to a calibrated custom-built pressure gauge. Wound pressures were recorded with no interface dressing and after the interposition of 4 layers of paraffin gauze dressings.

\section{Validation of the Accuracy of MRI Flow Sequences}

The sequence used for real-time MRI flow quantification in the present study is new and has not previously been validated. Therefore, validation was undertaken using a continuous-flow phantom employing 1-cm-diameter plastic tubing and tap water doped with $0.3 \mathrm{mg} / \mathrm{mL}$ gadolinium-based magnetic resonance contrast agent (Magnevist, gadopentetate dimeglumine, Schering Nordiska AB, Järfälla, Sweden). Accuracy was determined by comparing the MRI quantified flow with flow measured using beaker and timer at different flow rates. The sequence used for the established method of MRI flow quantification in the present study has previously been validated. ${ }^{14}$

\section{Calculations and Statistics}

The experiments were performed on 6 pigs. To eliminate time effects, the sequence of applying the 3 different negative pressures $(75,125$, and $175 \mathrm{~mm} \mathrm{Hg}$ ) was varied between the animals using a 3-by-3 Latin square design. Statistical analysis was performed using the Mann-Whitney test when comparing 2 groups and the Kruskal-Wallis test with Dunn's test for multiple comparisons when comparing 3 or more groups. Flow sequence accuracy was determined using Bland-Altman analysis. Values are presented as means \pm the standard error of the mean (SEM) unless otherwise stated.

\section{Results}

\section{Cardiac Output Quantified With the Established Technique}

MRI quantification of aortic flow was performed immediately before the negative pressure was turned on ( 0 minutes) and then $0.5,1,1.5,2,2.5$, and 3 minutes after application of negative pressure. The result showed that the cardiac output and stroke volume decreased immediately (Table 1 and Figure 2). After the initial decrease, only slight changes in cardiac output and stroke volume could be observed during the remaining 3 minutes of negative pressure. The decrease in cardiac output and stroke volume was followed by a successive increase in heart rate during the first 3 minutes, although this change was not significant (Figure 2). Immediately after switching the VAC off, the cardiac output, stroke volume, and heart rate returned to baseline values (Figure 2).

Similar patterns of hemodynamic changes were seen both in the absence and in the presence of 4 layers of paraffin gauze dressing over the heart. Interestingly, the magnitude of the hemodynamic effects by VAC was reduced in the presence of the paraffin gauze dressing (Table 1 and Figure 2). The effects of VAC hemodynamics were 
TABLE 1. Cardiac output and stroke volume reduction after VAC application in the presence and absence of a wound interface dressing

\begin{tabular}{|c|c|c|c|c|c|c|}
\hline & \multicolumn{3}{|c|}{ Cardiac output } & \multicolumn{3}{|c|}{ Stroke volume } \\
\hline & Without dressing & With dressing & $\boldsymbol{P}$ & Without dressing & With dressing & $\boldsymbol{P}$ \\
\hline$-75 \mathrm{~mm} \mathrm{Hg}$ & $-11.6 \% \pm 0.8 \%$ & $-7.7 \% \pm 0.7 \%$ & $<.01$ & $-10.7 \% \pm 1.8 \%$ & $-8.7 \% \pm .1 .0 \%$ & NS \\
\hline$-125 \mathrm{~mm} \mathrm{Hg}$ & $-12.8 \% \pm 1.3 \%$ & $-9.1 \% \pm 1.1 \%$ & NS & $-12.5 \% \pm 2.1 \%$ & $-10.8 \% \pm 1.6 \%$ & NS \\
\hline$-175 \mathrm{~mm} \mathrm{Hg}$ & $-15.4 \% \pm 0.8 \%$ & $-6.5 \% \pm 0.5 \%$ & $<.001$ & $-16.5 \% \pm 1.6 \%$ & $-8.6 \% \pm 0.8 \%$ & $<.01$ \\
\hline$P$ & NS & NS & & NS & NS & \\
\hline Mean & $-13.3 \% \pm 1.1 \%$ & $-7.8 \% \pm 0.9 \%$ & $<.001$ & $-13.2 \% \pm 2.0 \%$ & $-9.4 \% \pm 1.2 \%$ & $<.01$ \\
\hline
\end{tabular}

Mean values for each pressure $(-75,-125$, and $-175 \mathrm{~mm} \mathrm{Hg})$ were calculated from the measurements obtained immediately and during the first 3 minutes after application of negative pressure. Values are presented as mean reduction from baseline \pm SEM of 6 experiments. Note how the cardiac output reduction is not as pronounced in the presence of an interface dressing.

similar for the 3 negative pressures studied $(75,125$, and $175 \mathrm{~mm} \mathrm{Hg}$ ), with a nonsignificant tendency toward being more pronounced at higher negative pressures (Table 1 and Figure 2).
The mean baseline values were calculated from all 6 pigs, before negative pressure application, and were $2.9 \pm$ $0.1 \mathrm{~L} / \mathrm{min}$ for cardiac output, $34 \pm 1 \mathrm{~mL}$ for stroke volume, and $89 \pm 2$ beats per minute for heart rate.
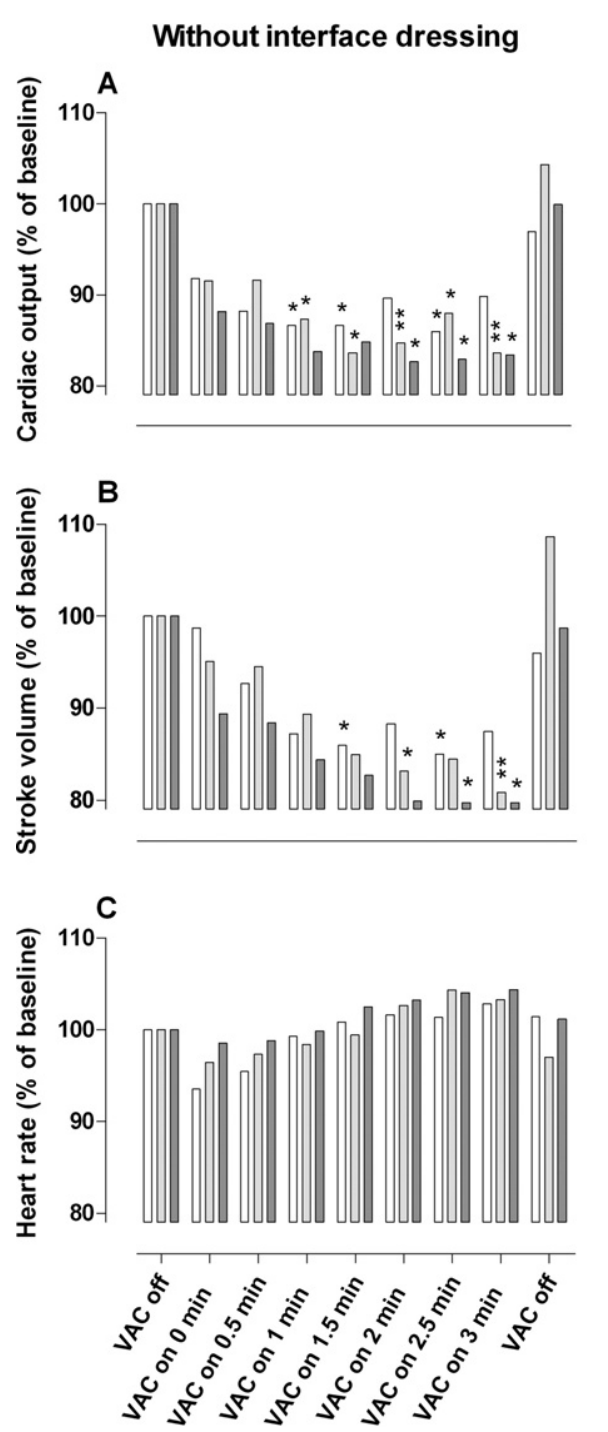
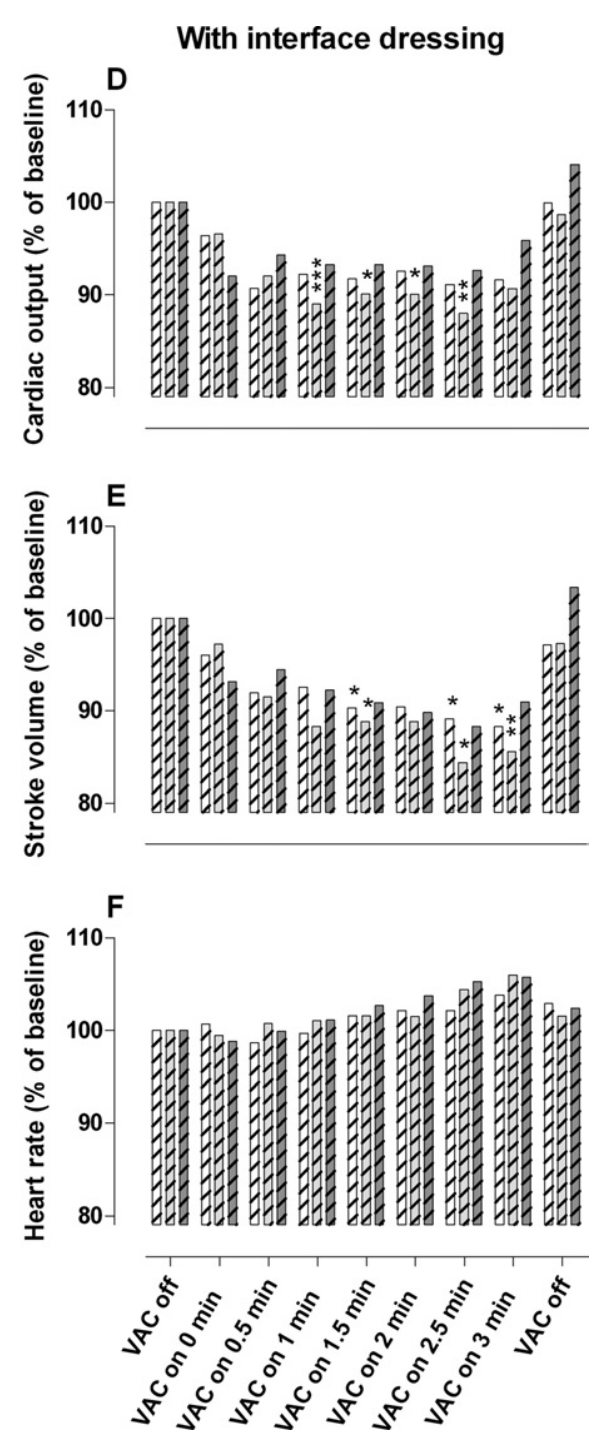

Figure 2. Cardiac output (A and D), stroke volume ( $B$ and $E$ ), and heart rate ( $C$ and $F$ ) were measured using the established technique for MRI flow quantification. Measurements were performed before the vacuum source was turned on (VAC off) and at a negative pressure of $75 \mathrm{~mm} \mathrm{Hg}$ (white bars), $125 \mathrm{~mm} \mathrm{Hg}$ (light gray bars), and $175 \mathrm{~mm} \mathrm{Hg}$ (dark gray bars), and 0, 0.5, $1,1.5,2,2.5$ and 3 minutes after the vacuum source was turned on (VAC on). Measurements were undertaken both with (A, B, and $C)$ and without (D, $E$, and F) 4 layers of paraffin gauze dressing covering the heart. The results are shown as means of 6 experiments. ${ }^{*} P<.05,{ }^{*} P<.01$, and ${ }^{* * *} P<$ .001. Note how cardiac output and stroke volume decrease over time. 

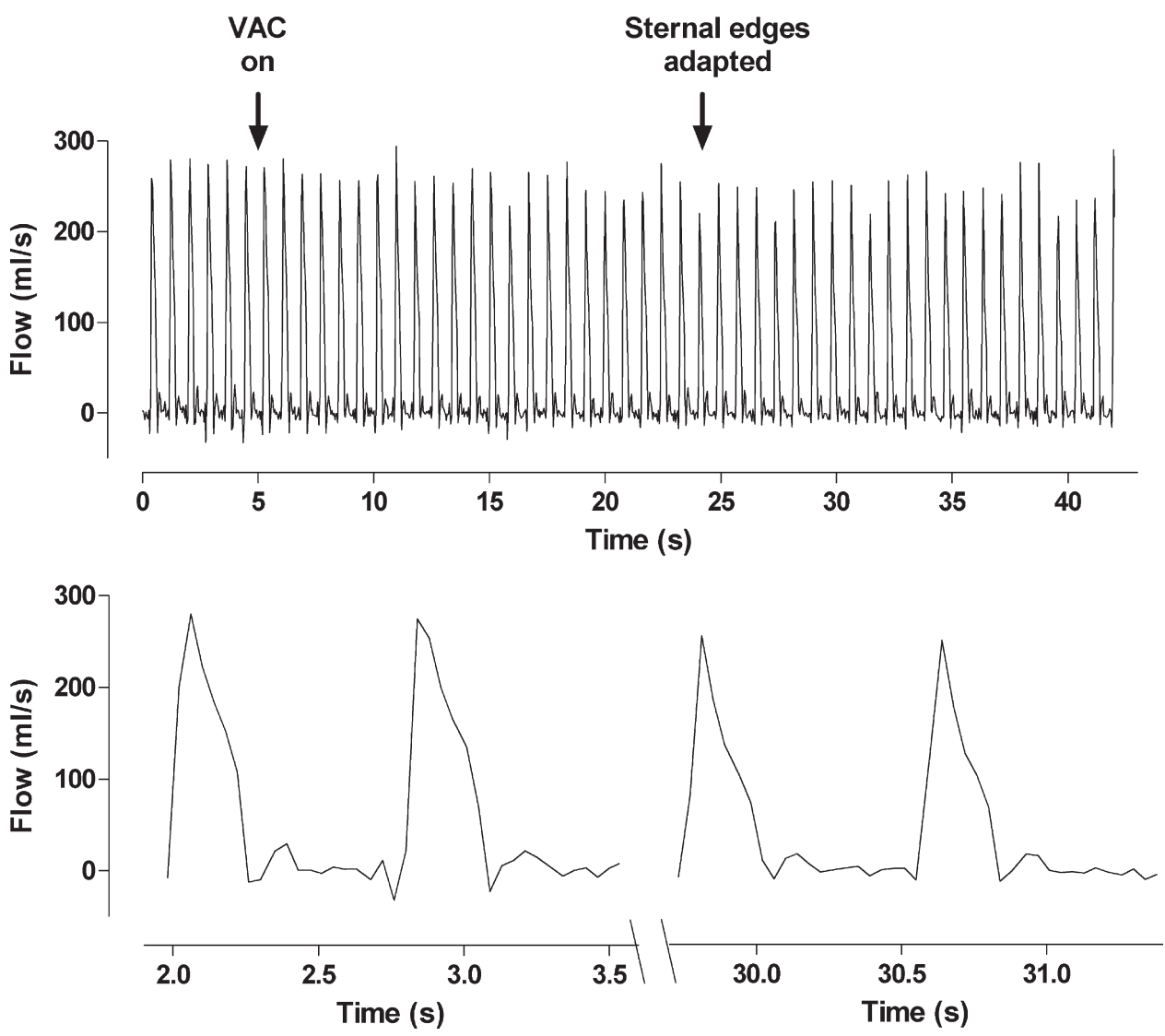

Figure 3. Results from one experiment during the application of $-175 \mathrm{~mm} \mathrm{Hg}$ in a sternotomy wound without interface dressing. Real-time MRI flow quantification in the ascending aorta throughout 42 seconds of the application of VAC (top panel). VAC on denotes when the vacuum source was turned on, and sternal edges adapted denotes when the lateral movement of the sternal edges was completed, which presumably reflects the completion of VAC application. (Bottom panel) A magnification of the flow in the top panel during representative heart beats. The first 2 beats (duration $=1.5$ seconds) show representative flow prior to the application of VAC. The second 2 beats (duration $=1.5$ seconds) show representative flow after the completion of VAC application, showing a reduced area under the curve compared with the bottom left panel, indicating a reduced stroke volume. These data are the basis for the results presented in Figure 4.

\section{Cardiac Output Quantified With the Real-time}

Technique

Real-time flow experiments, in which aortic flow was continuously quantified over 42 seconds, were performed to illustrate the initial time course of hemodynamic events with maximal temporal resolution. Stroke volume and cardiac output declined during the first 20 seconds of VAC application and then reached a steady-state level (Figures 3 and 4). Visual assessment of the magnetic resonance images during vacuum application showed that the sternal edges moved laterally and were drawn together during the initial 20 seconds and were stable thereafter. Twenty seconds after the application of VAC thereby presumably reflects the time point when the negative pressure had reached a steady state in the sternotomy wound. The heart rate did not seem to change in response to VAC application during these initial 42 seconds.

\section{Validation of Real-time Flow Quantification}

The mean error \pm standard deviation of the novel real-time MRI flow technique compared with flow measured by beaker and timer in a continuous flow phantom was $0.7 \% \pm$ $3.0 \%$, indicating high accuracy and low variability of the method.

\section{Left Ventricular Volumes}

MRI was used to quantify the left ventricular volumes. Images were acquired 1.5 minutes after the onset of VAC. Negative pressure induced a decrease in end diastolic volume $(57 \pm 7 \mathrm{~mL}$ before and $52 \pm 6 \mathrm{~mL}$ after application of 


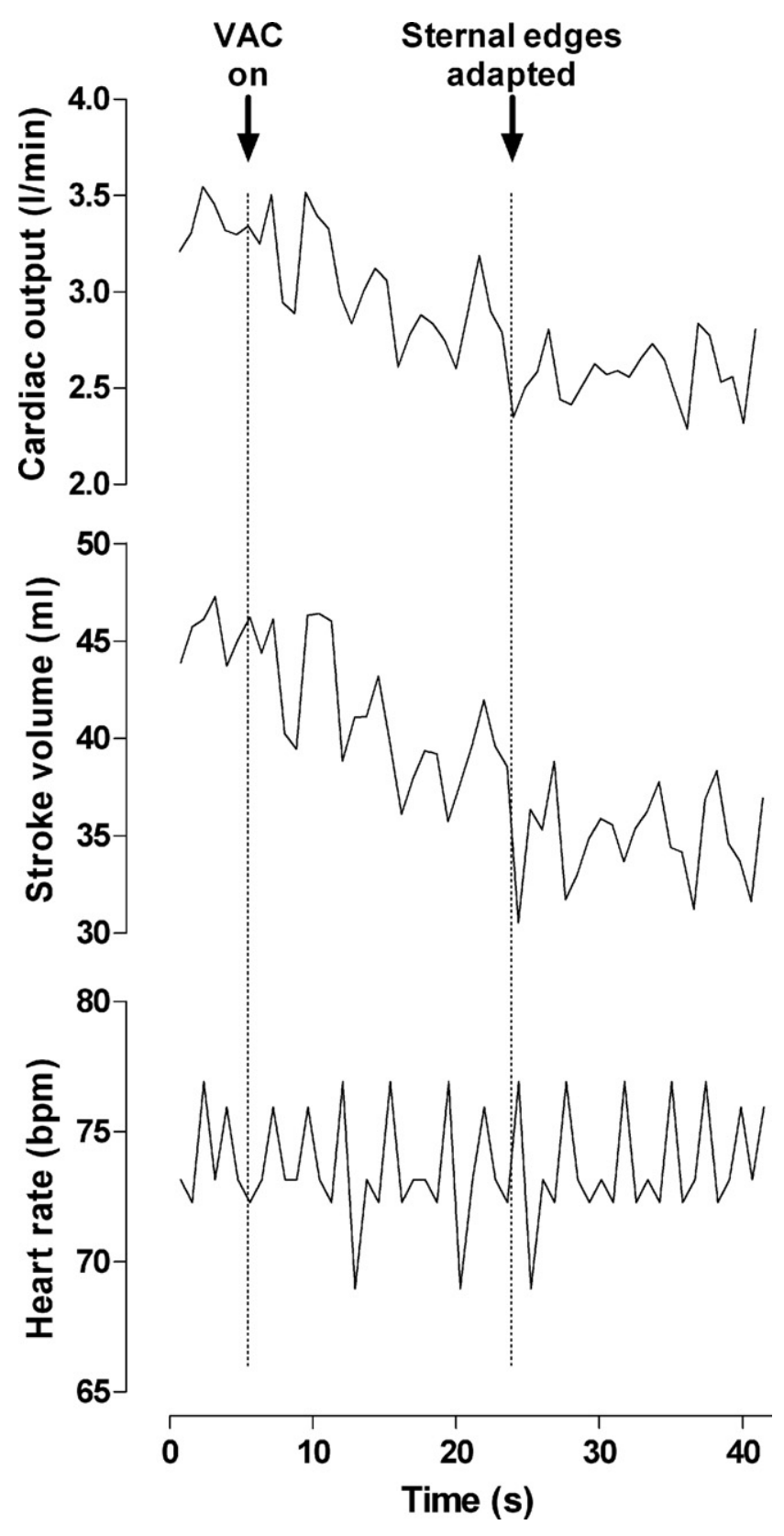

Figure 4. Changes in cardiac output (top), stroke volume (middle), and heart rate (bottom) measured using real-time MRI flow quantification during the application of $-175 \mathrm{~mm} \mathrm{Hg}$ in a sternotomy wound without interface dressing. VAC on denotes when the vacuum source was turned on, and sternal edges adapted denotes when the lateral movement of the sternal edges was completed, which presumably reflects the completion of VAC application. Note how the cardiac output and stroke volume declined during the application of negative pressure and then seemed to stabilize. See Methods for further details.
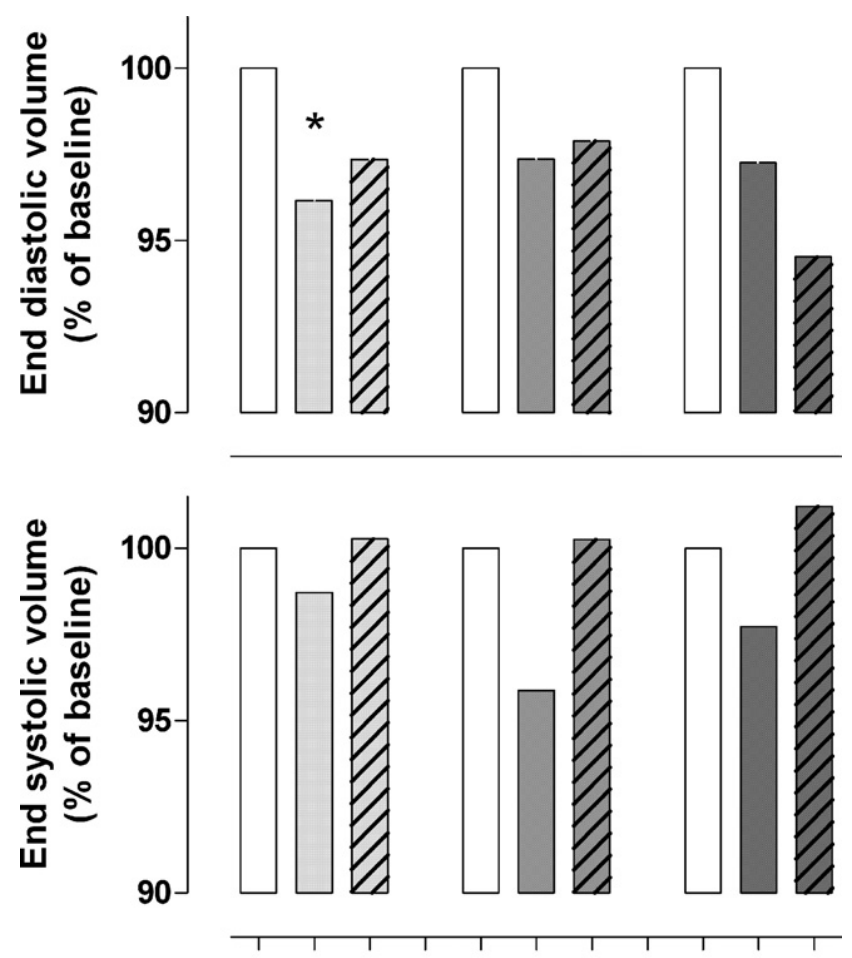

$75 \mathrm{~mm} \mathrm{Hg}$ in the absence of paraffin gauze dressing; $P<$ .05 ; Figure 5).

Wound Negative Pressure Level

The delivery of negative pressure was influenced by the presence of a paraffin gauze interface dressing. Pressures recorded in the space between the heart and the wound interface dressing were $22 \pm 3 \mathrm{~mm} \mathrm{Hg}$ lower than those set on the vacuum source. Interposition of 4 layers of paraffin gauze dressing over the heart increased the pressure difference between the wound space and the vacuum source to $53 \pm 5 \mathrm{~mm} \mathrm{Hg}$. For results, see Figure 6 . 


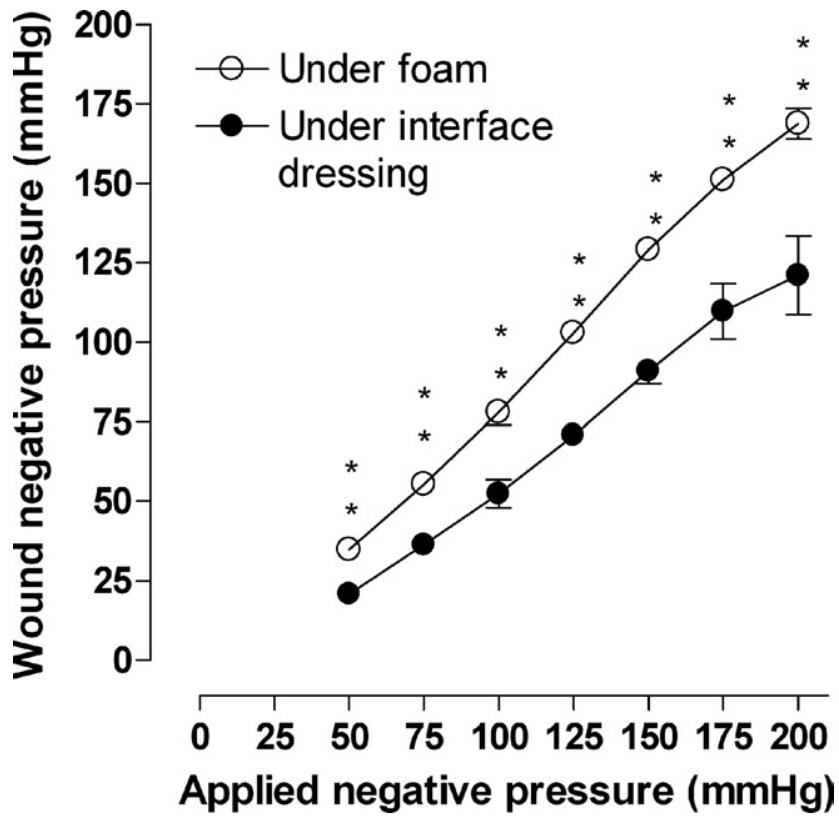

Figure 6. The vacuum source was set to successively deliver negative pressures from 50 to $200 \mathrm{~mm} \mathrm{Hg}$ at $25 \mathrm{~mm} \mathrm{Hg}$ increments. Wound pressures were recorded with no interface dressing (under foam) and after the interposition of 4 layers of the paraffin gauze dressings (under interface dressing). Values are presented as means \pm SEM from 6 experiments. ${ }^{* *} P<.01$. Note how the pressure in the wound was lower in the presence of an interface dressing.

\section{Discussion}

The effect of subatmospheric pressure on central hemodynamics is of major concern in cardiac surgery, especially because many patients with poststernotomy mediastinitis have reduced cardiac function. The present study aimed to quantify cardiac output and left ventricular volumes using MRI, which is known to be one of the most accurate methods for such measurements. ${ }^{15}$

\section{Hemodynamic Effects}

MRI indicated that after application of negative pressure, cardiac output, left ventricular end-diastolic volume, and stroke volume were reduced. Venous return to the heart is equal to cardiac output, and one might therefore expect that a more negative intrathoracic pressure induced by VAC would increase venous return and consequently cardiac output. However, our results showed that both cardiac output and left ventricular end-diastolic volume decreased when VAC was applied. This implies that VAC does not appear to induce an increase in venous return. Rather, VAC appears to exert a direct limiting effect on cardiac pumping, possibly by mechanically impairing the diastolic filling of the ventricles. VAC may cause a change in the intrathoracic anat- omy, which leads to a reduction in end-diastolic volume, stroke volume, and cardiac output. Intraventricular pressure waveforms for the right and left ventricles may provide more detailed analysis of the hemodynamic effects of VAC; however, such data were not acquired in the present study.

Real-time flow quantification was performed to achieve maximal temporal resolution for studying the initial hemodynamic changes due to VAC. The present results indicated that the decrease in cardiac output occurred during the first 20 seconds of VAC application, which implies that the hemodynamic changes are instantaneous and then seem to stabilize.

Previous studies of hemodynamic changes induced by VAC have shown conflicting results. Sonometry indicated decreased cardiac output, and thermodilution indicated unchanged or increased cardiac output. ${ }^{7,8}$ Both these methods have limitations. Sonometric assessment of left ventricular volumes involves two-dimensional measurements of distances, and the calculation of left-ventricular volumes is based on geometric assumptions. ${ }^{16}$ Moreover, thermodilution exhibits considerable variability between measurements. ${ }^{17}$ For the present study, MRI was used, and it is established as the in vivo reference standard ${ }^{9}$ for both assessment of flow in large vessels and quantification of chamber volumes. MRI has been independently shown to be highly accurate for measuring flow ${ }^{18}$ and to have equal accuracy and lesser variability compared with both thermodilution ${ }^{19}$ and the Fick method. ${ }^{20}$ For volume measurements, MRI was chosen because it offers true three-dimensional anatomic coverage and can be performed in any imaging plane orientation. ${ }^{21}$

Nevertheless, the sonometric method has less variability than the thermodilution technique. ${ }^{17,22}$ In the sonometric study by Conquest and colleagues, ${ }^{7}$ the cardiac output was found to be decreased by approximately $30 \%$. Our results show a less pronounced decrease of $9 \%$ reduction in cardiac output at the clinically accepted pressure of $-125 \mathrm{~mm} \mathrm{Hg}$ and with the commonly used paraffin gauze dressing covering the heart. Taken together, our findings suggest that a reduction in cardiac output occurs, but it is smaller than the reduction previously reported.

\section{Different Levels of Negative Pressure}

We chose to study the hemodynamic effects at $-75,-125$, and $-175 \mathrm{~mm} \mathrm{Hg}$. The pressure of choice for the clinical treatment of poststernotomy mediastinitis is $-125 \mathrm{~mm} \mathrm{Hg} .{ }^{10}$ Positive effects on blood flow to the peristernal thoracic wall $^{23}$ and biochemical pathways involved in granulation tissue formation ${ }^{24}$ have been observed at lower pressures $(-75$ to $-100 \mathrm{~mm} \mathrm{Hg})$. Greater pressures are used for larger cavities and traumatic wounds, ${ }^{25}$ and therefore the effects at $-175 \mathrm{~mm} \mathrm{Hg}$ were also investigated. The 3 negative pressures examined $(75,125$, and $175 \mathrm{~mm} \mathrm{Hg})$ all 
altered central hemodynamics. There was a tendency toward a more pronounced effect at high negative pressures, but these differences were not statistically significant.

The current study has shown that the VAC-mediated effects on central hemodynamics are not of a large magnitude regardless of negative pressure levels. The results from this study suggest that the level of negative pressure for clinical VAC therapy can most probably be selected on the basis of appropriateness for the wound and without particular consideration for potentially negative hemodynamic effects. The hemodynamic effect of intermittent negative pressure was not assessed in the current study because it is not believed to provide a satisfactory sternum stabilizing effect in the clinical setting.

\section{Wound Interface Dressing}

Wound interface dressings are commonly placed in the sternotomy wound to protect the right ventricle, lung tissue, and bypass grafts from the sternal edges. ${ }^{10}$ Furthermore, this is performed to prevent the formation of adherence between the heart and sternal edges and to facilitate the changing of dressings. One aim of the present study was to examine whether a paraffin gauze dressing would influence the effects of VAC on central hemodynamics. Interestingly, the magnitude of the negative hemodynamic effects by VAC was reduced in the presence of the dressing. Likewise, Conquest and colleagues ${ }^{7}$ applied a rectus muscle flap to the sternal wound prior to VAC therapy and showed a reduction in the impairment of central hemodynamics. A limitation of the current study is that hemodynamic assessment in the presence of a paraffin gauze dressing was always performed first, followed by hemodynamic assessment in the absence of the paraffin gauze dressing. This approach was chosen to minimize surgical manipulation of the sternal wound between MRI measurements.

The mechanism of protection provided by the paraffin gauze dressing cannot be deduced from the present study. Upon the delivery of negative pressure, the anterior portion of the right ventricle is sucked up toward the anterior thoracic wall and bulges into the space between the sternal edges, which deforms the anterior portion of the heart (data not shown). Hindering the delivery of topical negative pressure by an interface dressing may thereby lessen the deformation of the heart and the negative hemodynamic effects of VAC. Indeed, interface dressings have been shown to prevent the delivery of topical negative pressure. ${ }^{26}$ In the present study, 4 layers of paraffin gauze dressing impaired the delivery of negative pressure by an average of $31 \mathrm{~mm} \mathrm{Hg}$, depending on the level of negative pressure. Multiple layers of gauze may be advantageous as this approach provides a more robust mechanical barrier. We used Jelonet (Smith \& Nephew, Mölndal, Sweden), because this product has had satisfactory effects in the clinical setting at our center. We did not experience any problems with the Jelonet dressing coiling up or being dislocated into the thoracic cavity. Presumably, other paraffin gauze dressings (e.g. Mepitel, Mölnlycke Health Care, Göteborg, Sweden) might have similar effects.

\section{Implications for Invasive Hemodynamic Monitoring} Many patients with deep sternal wound infections have impaired cardiac function and heart failure due to ischemic heart disease. The ability to compensate for a decreased cardiac output due to VAC therapy may thereby be limited. It has been suggested that hemodynamic function should be carefully monitored in patients undergoing sternal VAC therapy. ${ }^{7}$ The current study assessed only the first 3 minutes of VAC therapy and, after the onset of vacuum, cardiac output was reduced immediately and then stabilized at the lower level. Conquest and associates ${ }^{7}$ also reported an initial hemodynamic effect that remained unaltered throughout 60 minutes of therapy. This supports the notion that hemodynamic effects remain unchanged after the initial reduction. Studies of the chronic nature of the hemodynamic effects must be performed before recommendations about invasive monitoring can be made. Ideally, these studies should be carried out in a model of heart failure in which the physiologic compensatory mechanisms are compromised.

\section{Conclusions}

VAC resulted in an immediate decrease in cardiac output and stroke volume. The left ventricular end-diastolic volume also decreased, which implies that VAC may have a direct limiting effect on diastolic filling. Interposition of a wound interface dressing lessened the hemodynamic effects of VAC therapy. Interestingly, in the commonly used clinical setting with a VAC treatment at $-125 \mathrm{~mm} \mathrm{Hg}$ combined with an interface dressing covering the heart, the hemodynamic impairment is of a much lesser magnitude (9\% decrease in cardiac output) than that previously reported $(30 \%)^{7,12}$

We thank Dr Onnen Grauhan and Dr Thomas Krabatsch for their valuable comments on the manuscript.

\section{References}

1. El Oakley RM, Wright JE. Postoperative mediastinitis: classification and management. Ann Thorac Surg. 1996;61:1030-6.

2. Raudat CW, Pagel J, Woodhall D, et al. Early intervention and aggressive management of infected median sternotomy incision: a review of 2242 open-heart procedures. Am Surg. 1997;63:238-41; discussion 241-2.

3. Crabtree TD, Codd JE, Fraser VJ, et al. Multivariate analysis of risk factors for deep and superficial sternal infection after coronary artery bypass grafting at a tertiary care medical center. Semin Thorac Cardiovasc Surg. 2004;16:53-61.

4. Lu JC, Grayson AD, Jha P, et al. Risk factors for sternal wound infection and mid-term survival following coronary artery bypass surgery. Eur J Cardiothorac Surg. 2003;23:943-9. 
5. Obdeijn MC, de Lange MY, Lichtendahl DH, de Boer WJ. Vacuumassisted closure in the treatment of poststernotomy mediastinitis. Ann Thorac Surg. 1999;68:2358-60.

6. Sjogren J, Gustafsson R, Nilsson J, et al. Clinical outcome after poststernotomy mediastinitis: vacuum-assisted closure versus conventional treatment. Ann Thorac Surg. 2005;79:2049-55.

7. Conquest AM, Garofalo JH, Maziarz DM, et al. Hemodynamic effects of the vacuum-assisted closure device on open mediastinal wounds. J Surg Res. 2003;115:209-13.

8. Sjögren J, Gustafsson R, Wackenfors A, et al. Effects of vacuumassisted closure on central hemodynamics in a sternotomy wound model. Interact CardioVasc Thorac Surg. 2004;3:666-71.

9. Pennell DJ, Sechtem UP, Higgins CB, et al. Clinical indications for cardiovascular magnetic resonance (CMR): Consensus Panel report. Eur Heart J. 2004;25:1940-65.

10. Gustafsson RI, Sjogren J, Ingemansson R. Deep sternal wound infection: a sternal-sparing technique with vacuum-assisted closure therapy. Ann Thorac Surg. 2003;76:2048-53; discussion 2053.

11. Institute of Laboratory Animal Research Commission on Life Sciences NRC. Guide for the care and use of laboratory animals. Washington, DC: National Academy Press; 1996.

12. Petzina R, Gustafsson L, Mokhtari A, et al. Effect of vacuum-assisted closure on blood flow in the peristernal thoracic wall after internal mammary artery harvesting. Eur J Cardiothorac Surg. 2006;30:85-9.

13. Pennell DJ. Ventricular volume and mass by CMR. J Cardiovasc Magn Reson. 2002;4:507-13.

14. Beerbaum P, Korperich H, Gieseke J, et al. Blood flow quantification in adults by phase-contrast MRI combined with SENSE-a validation study. J Cardiovasc Magn Reson. 2005;7:361-9.

15. Arheden H, Stahlberg F. Blood flow measurements. In: Higgins CB, de Roos A, editors. MRI and CT of the Cardiovascular System, 2nd ed. New York, NY: Lippincott; 2006. p. 71-90.

16. Sonometrics Corporation. Sonometrics product information. Available from: http://sonometrics.com/applications_sonovconduct3.html.
17. Espersen K, Jensen EW, Rosenborg D, et al. Comparison of cardiac output measurement techniques: thermodilution, Doppler, CO2rebreathing and the direct Fick method. Acta Anaesthesiol Scand. 1995;39:245-51.

18. Arheden H, Holmqvist $C$, Thilen U, et al. Left-to-right cardiac shunts: comparison of measurements obtained with MR velocity mapping and with radionuclide angiography. Radiology. 1999;211:453-8.

19. Kuehne T, Yilmaz S, Schulze-Neick I, et al. Magnetic resonance imaging guided catheterisation for assessment of pulmonary vascular resistance: in vivo validation and clinical application in patients with pulmonary hypertension. Heart. 2005;91:1064-9.

20. Razavi R, Hill DL, Keevil SF, et al. Cardiac catheterisation guided by MRI in children and adults with congenital heart disease. Lancet. 2003;362:1877-82.

21. Bellenger NG, Davies LC, Francis JM, et al. Reduction in sample size for studies of remodeling in heart failure by the use of cardiovascular magnetic resonance. J Cardiovasc Magn Reson. 2000;2: 271-8.

22. Pascoe JR, Hiraga A, Hobo S, et al. Cardiac output measurements using sonomicrometer crystals on the left ventricle at rest and exercise. Equine Vet J Suppl. 1999;30:148-52.

23. Wackenfors A, Gustafsson R, Sjogren J, et al. Blood flow responses in the peristernal thoracic wall during vacuum-assisted closure therapy. Ann Thorac Surg. 2005;79:1724-30; discussion 1730-1.

24. Tang SY, Chen SZ, Hu ZH, et al. [Influence of vacuum-assisted closure technique on expression of Bcl-2 and NGF/NGFmRNA during wound healing]. Zhonghua Zheng Xing Wai Ke Za Zhi. 2004;20:139-42.

25. Argenta LC, Morykwas MJ. Vacuum-assisted closure: a new method for wound control and treatment: clinical experience. Ann Plast Surg. 1997;38:563-76; discussion 577.

26. Jones SM, Banwell PE, Shakespeare PG. Interface dressings influence the delivery of topical negative-pressure therapy. Plast Reconstr Surg. 2005;116:1023-8. 